\title{
CARBON ATOMIC WIRES: FROM STARS TO NANOTECHNOLOGY
}

\author{
CARLO S. CASARI (*)
}

Nota presentata dal s.c. Carlo Bottani

(Adunanza del 2 febbraio 2012)

SUNTO. - Il carbonio può presentarsi in una grande varietà di sistemi e nanostrutture di diversa dimensionalità come nel caso di fullereni, nano tubi e grafene, tutti con proprietà specifiche di grande interesse per la scienza di base e per quella applicata. In questo contesto atomi di carbonio con ibridazione di tipo $s p$ possono aggregarsi per formare fili atomici le cui proprietà risultano fortemente dipendenti dalle dimensioni. La ricerca del carbonio $s p$ ha una lunga storia che ha toccato anche l'astrofisica e i minerali trovati in crateri da impatto meteoritico e oggigiorno, anche se strutture a base di carbonio sp sono tuttora generalmente considerati sfuggenti e instabili, diversi sistemi nano-strutturati interamente costitutiti da (o contenenti) fili atomici di carbonio vengono prodotti e studiati. Così come il grafene viene considerato il materiale più sottile mai ottenuto (spessore di un atomo) i fili atomici di carbonio rappresentano veri sistemi mono-dimensionali (diametro di un atomo) con grandi potenzialità per le nanoscienze e le nanotecnologie. In questa Nota si discute lo stato attuale della ricerca sui fili atomici di carbonio, partendo dalla descrizione dei sistemi ideali come termine di paragone per strutture reali, allo scopo di mostrare come le proprietà possano essere modulate controllando la lunghezza (cioè il numero di atomi di carbonio) e il tipo di terminazione (atomi o gruppi molecolari o nanostrutture). Vengono presentate le principali tecniche di sintesi e le strategie per ottenere strutture stabili. Particolare enfasi viene messa nell'uso delle spettroscopie vibrazionali per ottenere informazioni sulle proprietà strutturali ed elettroniche, infine, vengono discusse le prospettive aperte per lo sviluppo di dispositivi innovativi basati sull'utilizzo di questi sistemi.

$* * *$

(*) Dipartimento di Energia, Politecnico di Milano, Italy.

E-mail: carlo.casari@polimi.it 
ABSTRACT. - Carbon can produce a wide variety of systems and nanostructures with different dimensionality as exemplified by fullerenes, nanotubes and graphene, all with peculiar properties of great interest for fundamental and applied research. In this framework $s p$-hybridized carbon atoms can arrange to form atomic wires with size dependent functional properties. The $s p$ form of carbon has a long story passing through astrophysics and minerals found in meteor craters and, although still considered elusive and unstable, nowadays a number of nano-systems constituted by or containing sp-carbon wires have been produced and characterized. As graphene is considered the thinnest material (one-atom thick), carbon atomic wires represent true 1-dimensional systems (one- atom diameter) with a great potential for nanoscience and nanotechnology. The status of research on carbon atomic wires is here discussed, starting from the description of ideal systems to real structures, showing that the properties can be tuned by controlling the wire length (i.e. number of carbon atoms) and termination (atom, molecular group or nanostructure). Synthesis techniques are presented as well as strategies to have stable wires. A particular attention will be given to the use of vibrational spectroscopy to provide insight on the structure and electronic properties of these systems. Perspectives for novel devices based on the exploitation of the electronic properties of these systems are also discussed.

\section{INTRODUCTION}

Carbon is at the origin of life, it is a versatile element since it can arrange its valence electrons in different ways to produce a wide variety of molecules and structures. This is clear in organic chemistry where the three possible $\mathrm{sp}^{3}, \mathrm{sp}^{2}$, and $\mathrm{sp}^{1}$ hybridizations lead to alkanes (e.g. methane), alkenes (e.g. ethylene) and alkynes (e.g. acetylene) molecules, respectively. The different geometry of the hybridized orbitals results in linear (1-dimensional), planar (2-dimensional) or tetrahedral (3-dimensional) spatial organization. In astrophysics the presence of short linear carbon molecules has been detected in the interstellar medium and experimental studies to understand their formation drove the discovery of fullerenes. Moving to solid state systems, carbon shows only two allotropes: graphite and diamond corresponding to $\mathrm{sp}^{2}$ and $\mathrm{sp}^{3}$ hybridization. The lack of a third crystalline form based on $\mathrm{sp}^{1}$ hybridization (linear carbon or carbyne) has attracted the attention of scientist for the last two centuries. The discovery of this new carbon was claimed in mineral samples found in a meteor crater, nevertheless the existence of such a new pure carbon solid has still today to be confirmed and the efforts in this direction have been progressively reduced. In this context the advent of nanotechnology and the great impact of the discovery of carbon nanostructures such as fullerenes, nanotubes and graphene have 
raised a renewed interest in nanoscale carbon systems with linear structures.[1] Carbon nanostructures can be classified according to their hybridization, as shown in Fig. 1. Graphene, one isolated honeycomb layer of graphite, is a 2-dimensional structure that can be placed at the $\mathrm{sp}^{2}$ vertex. Nanotubes and fullerenes are curved structures that can be put in the pathway from $\mathrm{sp}^{2}$ to $\mathrm{sp}^{3}$ where the $\mathrm{sp}^{3}$ character increases for smaller structures (the fullerene $\mathrm{C}_{20}$ has no hexagons in its structure). Finally ending at the $\mathrm{sp}^{3}$ vertex with nanodiamond. Moving from $\mathrm{sp}^{2}$ to $\mathrm{sp}^{1}$ we can find a mixed $\mathrm{sp}^{2}-\mathrm{sp}^{1}$ planar structure (graphyne), ring structures and linear 1-dimensional atomic chains. Approaching $\mathrm{sp}^{1}$ from $\mathrm{sp}^{3}$ we find hypothetical possible mixed systems sometimes called superdiamond or sp-sp ${ }^{3}$-yne-diamond.

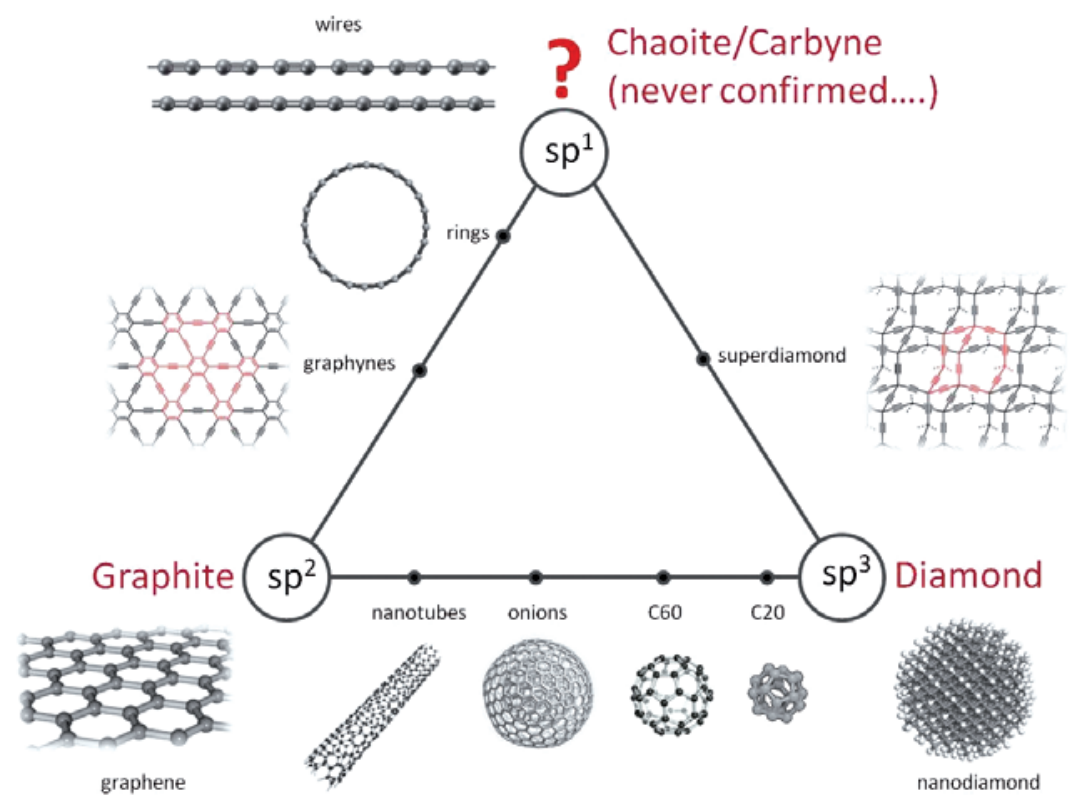

Fig. 1 - Three phase diagram showing possible classification of carbon nanostructures according to the bybridization state of carbon atoms.

Carbon atomic wires possess a large conjugation because of the two $\mathrm{p}$ orbitals provided by each atom. This gives rise to a strong structure-property relationship that makes these systems very appealing for 
fundamental and applied research. Ideal wires are infinite long systems and present only two possible geometric configurations: an alternated structure with single and triple bonds, also called polyyne and an equalized structure with a sequence of double bonds, also named cumulene (see Fig. 2). As discussed in section 3, cumulene is a 1-dimensional metal while polyyne is a semiconductor. In real systems the wire has a finite length and a defined termination. As a consequence the electronic (metal or semiconductor) and optical properties (optical absorption) are determined by the size and the type of termination. The control of wire structure can open the way to novel functional systems to be used as building blocks for advanced materials with tunable functionality. Of course, one needs to know how to fabricate wires, how to characterize their structure and functional properties before being able to make devices and there are still some important challenges to this goal. Here the present status of research about carbon atomic wires is presented to discuss the potentialities of these systems as advanced materials for future technological applications.

\section{A BRIEF HISTORY OF SP-HYBRIDIZED CARBON}

Graphite and diamond are known since the ancient time, but it was in 1796 that S. Tennant experimentally demonstrated that diamond and graphite were made of the same substance.[2] At the end of nineteenth's century some works were made trying to synthesize linear or polymeric forms of carbon by Glaser in 1870 and Baeyer in 1885. As a consequence of Baeyer's theories stating the impossibility to produce linear carbon the research on sp-carbon suffered a strong damping.

A new impulse was given in the sixties by claims of discovery of a natural form (a new allotrope) of carbon with linear structure. The first paper claiming this discovery (named carbyne, while the mineral form was called chaoite) dates back to the sixties when El Goresy and Donnay while analyzing by $\mathrm{x}$-ray diffraction graphite-rich material coming from a meteor impact crater (the Noerdlinger Ries crater in Germany) found a primitive cell size different from graphite and other carbon-based materials. [3] Other authors started to investigate the conditions for the formation of this material and the related position in the phase diagram of carbon.[4,5] In the same period Russian scientists reported the synthesis of carbyne. Kudryavtsev and coworkers registered the discovery 
of carbyne (in russian, see e.g. [6]), a sort of patent issued by the USSR Committee of Inventions and Discoveries. In the eighties strong criticisms were raised for example by Smith and Buseck about the interpretation of the diffraction results and the existence of carbyne was object of intense debates, as witnessed by the papers appeared on Science with titles like 'Carbyne: do they really exist?'.[7-9] In the meantime the search for sp carbon linear clusters to understand some spectral features observed in the interstellar medium was a hot topic in astrophysics. Researcher were trying to reproduce in laboratory the conditions for carbon cluster formation in the stars, including Sir H. Kroto at the University of Sussex in UK. He was trying to synthesize $\mathrm{HC}_{5} \mathrm{~N}$ and longer polyyne-like systems by laser vaporization source when in 1985, together with Richard Smalley and Robert Curl, observed the presence of large clusters made by 60 carbon atoms ( 20 hexagons and 12 pentagons forming a soccer ball). In 1996 Kroto, Smalley and Curl were awarded with the Nobel Prize in Chemistry 'for their discovery of fullerenes'; the story of the discovery is nicely reported in the Nobel Lecture given by Kroto.[10]

In 1995 R. Lagow and coworkers reported the synthesis of 'liner acetylenic carbon' up to 28 carbon atoms.[11] They optimistically stated that systems of more than 300 atoms were potentially possible to be synthesized. At present, the occurrence of sp-hybridized carbon has been observed in many carbon-based materials and structures, embedded in cold gas matrices,[12] in free carbon clusters in the gas phase, $[13,14]$ in pure sp-sp ${ }^{2}$ systems,[15] in liquids,[16] inside carbon nanotubes (CNTs) [17] and connecting graphene sheets.[18] Nowadays, polyynes in liquids (even number of carbon atoms up to 20) are synthesized even as size-selected samples [19-21] and with well defined end groups.[22] Solid state samples have been also produced [23] and recently R.R. Tykwinsky and co-workers reported the synthesis of chains up to 44 carbon atoms terminated by bulky groups.[24] The latter system is stable in air and at room temperature in the form of a solid sample. Tykwinski reported also the synthesis of long cumulenes [25] even though they seem more difficult to produce and a few works are present reporting their observation. By playing with termination-induced electronic arrangement, short cumulenic structures can be produced, as reported by Cataldo [26] and extensively discussed in the review by Cadierno et al..[27] Both cumulenes and polyynes were detected in a pure sp-sp ${ }^{2}$ cluster-assembled amorphous carbon sys- 
tem.[15,28-30] More recently reversible transformation of polyyne into cumulenes under UV irradiation has been also reported.[31]

\section{STRUCTURE AND PROPERTIES OF CARBON WIRES}

The ideal model of carbon atomic wire is an infinite chain of carbon atoms. In this case there are only two ways for carbon atoms to arrange in the wire: a sequence of double bonds (cumulene) and single and triple alternated bonds (polyyne), see Fig. 2. Cumulenes have one atom per unit cell and each contributes with two electrons, one for each $\pi$-orbital. This results in a half-filled conduction band and the system is a 1-dimensional metal. On the contrary, polyynes have two atoms per unit cell thus filling completely the valence band and leaving an empty conduction band. As a result polyyne is a 1-dimensional semiconductor. The same approach applies also for the vibrational properties (i.e. phonons). Cumulene is an example of a 1-dimensional homo-atomic chain usually used to describe the vibrational properties of more complex systems while polyyne is a homo-atomic chain with different bond lengths (and strength) that is the analogue of a hetero-atomic chain. Simple textbook calculations show that cumulene has only acoustic phonons while polyynes have acoustic and optic phonon branches. More detailed calculations lead to corrections in the optical phonon frequencies at the center of the Brillouin zone when conjugation effects are considered.
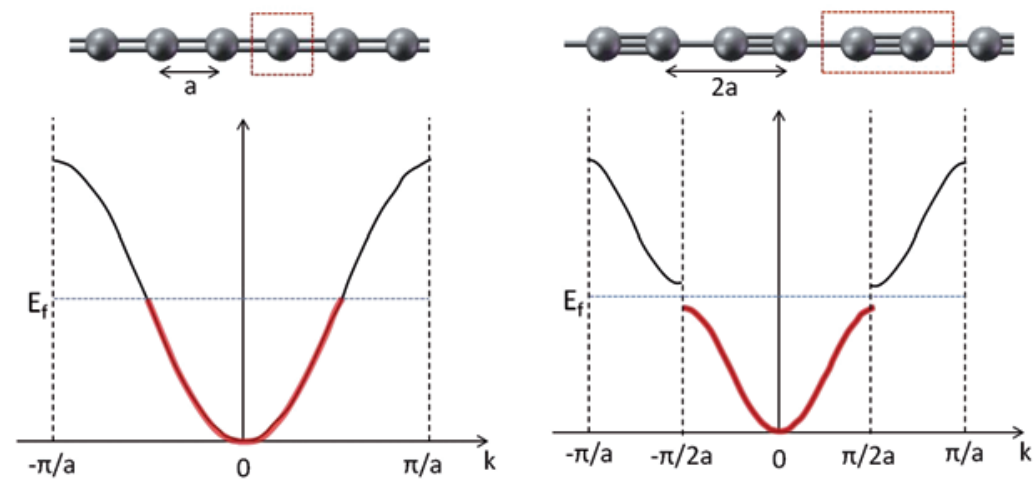

Fig. 2 - Schematic electronic band structure for infinite cumulene and polyyne showing the metal and semiconductive behavior. 
The link between the two possible wire structures is the onset of Peierl's distorsion, a theoretical prediction made by Peierl for 1-D metals.[32] According to Peierl a 1-D metal can undergo a metal-to insulator transition since a dimerized chain structure is energetically more favorable than an equalized one. The alternated bond structure (dimerized) is the result of a balance between the elastic strain introduced to distort the chain and the gain obtained by lowering the energy of electrons. In the case of carbon wires such instability would mean that cumulenes are unstable and tend to rearrange in polyyne form. This actually occurs only for the infinite wire while for real systems the role of Peierls distorsion is less important as discussed in the following.

The distinction of wires in the two discussed configurations, alternated and equalized chains, strictly holds only for ideal infinite systems, while for real finite wires the distinction between cumulenic and polyynic configuration is somehow relaxed since in both cases approaching the ends the bond lengths can change due to a different termination. The termination has usually a different bond length and this affects the entire wire structure so that the shorter the wire the larger is the terminating effect. A distribution of bond lengths along the wire is typically found when simulating the structure of finite and terminated wires. In this case the bond length alternation (BLA, the length difference between two adjacent bonds) is a more correct parameter to describe the wire structure. The ideal cumulene has $\mathrm{BLA}=0$ while simulations of the structure of real and finite cumulene-like systems show that the BLA is small but not zero. Fig. 3 shows a comparison of the BLA evaluated from first principles calculations for polyynes and cumulenes as a function of the wire length (i.e. number of carbon atoms).

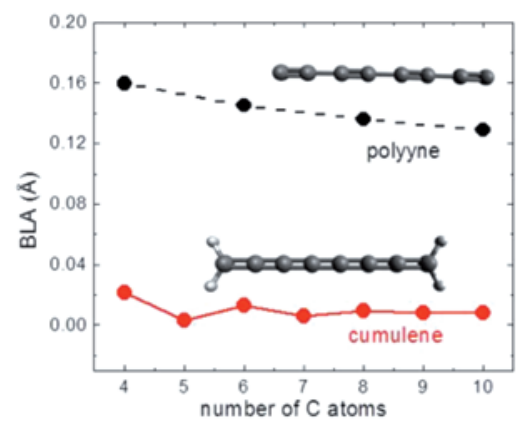

Fig. 3 - Dependence of bond length alternation (BLA) values for finite-length polyynes and cumulenes as a function of the number of carbon atoms. The values are obtained from first principles calculations. [47] 
In the same way the energy gap and the optical and transport properties depend not only on the type of structure but also on chain length (i.e. the number of carbon atoms) and type of end groups.[33-35] The electronic bands show a gap, which depends on the BLA value.[33] Although the electronic properties have been theoretically addressed by many authors, the experimental evidences are scarce. Only a few works reported the study of electronic core levels and the valence band properties by electron spectroscopy techniques.[13,36,37]

Vibrational properties show a similar dependence in the phonon dispersion relation. In particular the frequency of the longitudinal optical phonons at the center of the Brillouin zone scales from 1800 to more than $2200 \mathrm{~cm}^{-1}$, depending on the wire structure, thus suggesting why vibrational spectroscopy techniques are extremely useful for the characterization of sp-carbon wires, as discussed in section 5 .

\section{SyNhthesis TECHNIQUeS}

Today, many techniques, both physical and chemical, have been demonstrated to produce sp carbon wires in different forms, mainly in a bottom-up approach.[19] Physical techniques are mainly based on the rapid quenching of a carbon vapour in different environments (see Fig. 4). Supersonic carbon cluster sources based on the arc discharge between graphite electrodes (i.e. pulsed microplasma cluster source PMCS developed by P. Milani) demonstrated the capability to produce sp-rich amorphous carbon films (estimated to be up to 40\%).[28] A similar approach was exploited using thermal or laser vaporization cluster sources.[12,38] Sp carbon has been also produced by ion irradiation of amorphous carbon [39] and by femtosecond (fs) laser irradiaton of a graphite target.[40] fs laser pulses have been used to produce amorphous carbon films containing $\mathrm{sp}, \mathrm{sp}^{2}$ and $\mathrm{sp}^{3}$ phase.[41] For all the above-mentioned techniques sp carbon is present in amorphous (mainly $\mathrm{sp}^{2}$ carbon moieties) and a fine control of the chain structure and length is still to be achieved. Isolated wires can be produced by laser ablation (with both fs and ns pulses) of carbon solid targets (graphite) or suspensions (fullerenes, diamond particles) in liquids and particularly in the polyyne-like form with an even number of carbon atoms.[16, 40] With reference to polyynes in solution an easy-to-use and costeffective technique is the submerged arc discharge in liquids developed by F. Cataldo.[42] This technique allows also controlling, to some 
extent, the chain termination by selecting suitable solvents.[43] As an example short cumulenic structures terminated by sulfur atoms have been produce by arcing in carbon sulfide.[26]

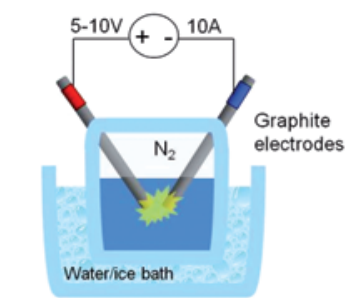

Submerged arc discharge

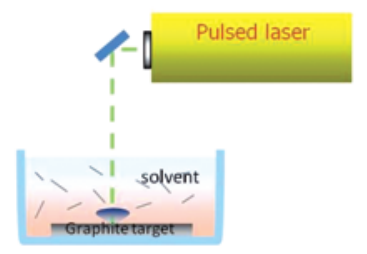

Pulsed laser ablation in liquid

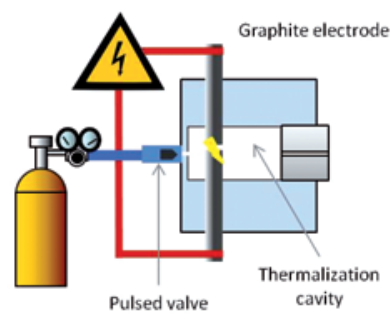

Pulsed Microplasma Cluster Source

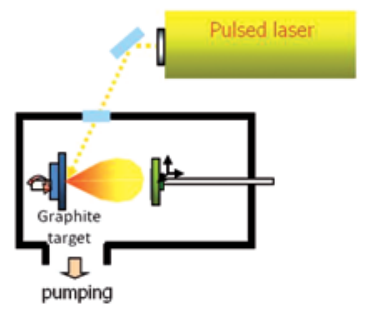

Pulsed laser deposition

Fig. 4 - Examples of physical techniques for the synthesis of carbon atomic wires

Different chemical techniques have been used to synthesize a large number of sp carbon chains terminated with different molecular groups as reported in the review by Gladysz et al..[22] Among the most employed chemical routes we cite the dehydropolycondensation of acetylene such as the Glaser reaction based on the oxidative coupling reaction of ethynyl groups by copper salts; polycondensation reactions of halides; dehydrohalogenation of polymers such as the chemical carbonization of poly(vinylidene halides) (PVDH). Tykwinski reported the synthesis of very long and stable polyynes and cumulenes.[25]

Top-down methods start from a different carbon structure that can be turned into a wire by means of mechanical stress, electric field or removal of selected atoms. One feasible possibility is represented by electron bombardment of a single graphene flake in a transmission electron microscope (TEM) thus allowing to selectively remove carbon atoms until a single atomic chain is connecting two separate graphene edges.[18] However the method is extremely time-consuming and requires the elec- 
tron beam and the image capabilities of a TEM. Such approach allows to obtain very nice TEM images of wires suspended between graphene edges even though the unambiguous identification of the chain structure was not possible. Some of the systems that can be produced by these techniques are reported in Fig. 5.
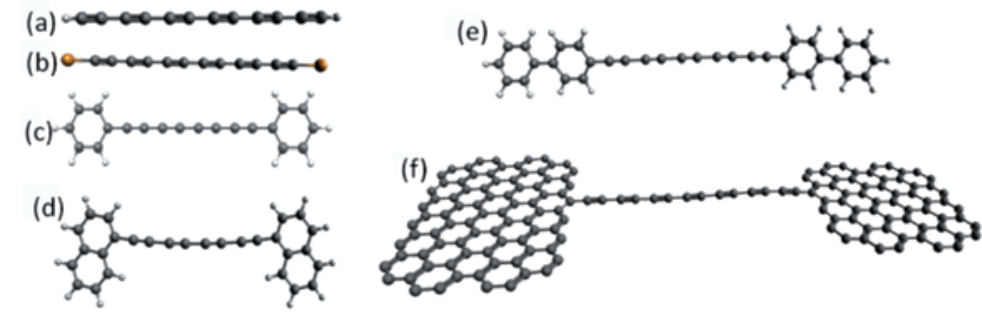

Fig. 5 - Structure of real wires with different terminations, from top to bottom (a to f) polyyne terminated by bydrogen, copper, phenyl, bis-phenyl, naphtyl and graphene.

\section{Characterization TECHNIQUES}

Vibrations in molecules and solids are strongly dependent on the atomic arrangement and chemical bond. In carbon nanostructures the local atomic organization can be very different due to the hybridization state and local order, hence a vibrational technique sensitive to the local vibrations can provide a wealth of structural and chemical information. Raman spectroscopy is a technique based on inelastic scattering of light by the thermally excited vibrations (i.e. phonons in quantum solid state approach) of the material. The technique is based on the analysis of the spectrum of an incident monochromatic light (laser light with a given wavelength) after the interaction with a sample. The outcoming light can have wavelengths higher and lower than the incident one. In a quantum particle description incident photons are inelastically scattered by phonons and come out from the material with a different energy.[44] The difference equals the energy exchanged in the process in creating or annihilating a phonon. In this way the spectrum contains information on the phonon energy that can be a fingerprint of some characteristics of the investigated material. Raman turns out to be extremely useful to investigate carbonbased materials and nanostructures as exemplified in Fig. 6. Different types of carbon nanostructures are characterized by Raman signatures that can be used to investigate the structural properties, a chief example being 
graphene for which the number of layers can be unambiguously detected by inspecting the Raman spectrum. $[45,46]$ Sp carbon shows Raman peaks in a specific spectral region $\left(1800-2200 \mathrm{~cm}^{-1}\right)$ where no other peaks from $\mathrm{sp}^{2}$ carbon (1300-1600 $\mathrm{cm}^{-1}$ and $2700-3200 \mathrm{~cm}^{-1}$ ) or from diamond (1332 $\mathrm{cm}^{-1}$ ) are present, thus permitting to identify its presence, even when other carbon phases coexist, as in the case of sp-sp ${ }^{2}$ carbon moieties. The Raman peak is associated to the carbon-carbon stretching mode and polyynes are expected to have higher Raman frequencies than cumulenes, permitting to be sensitive to the structural arrangement.[47] In addition the peak frequency decreases with increasing the chain length due to modulation of the BLA with the wire length and approaches the value of the ideal structure. In addition effects of chain conformations such as chain bending, torsions and different arrangement of the terminating groups and to what extent they affect the Raman spectrum have been studied both experimentally and theoretically.[21,48,49] This allows to get a lot of information by a detailed inspection of the Raman spectrum also with the support of theoretical simulations. As shown in Fig. 6 different types of polyynes display specific Raman spectra depending on wire length, termination and local order. For instance, from the analysis of the spectrum of phenyl-terminated polyynes it is possible to evidence the vibration of the phenyl ring (at about $1600 \mathrm{~cm}^{-1}$ ) and to associate the different peaks in the 2000-2200 $\mathrm{cm}^{-1}$ region to the contribution of wires of different length from 4 to 12 carbon atoms. [48]
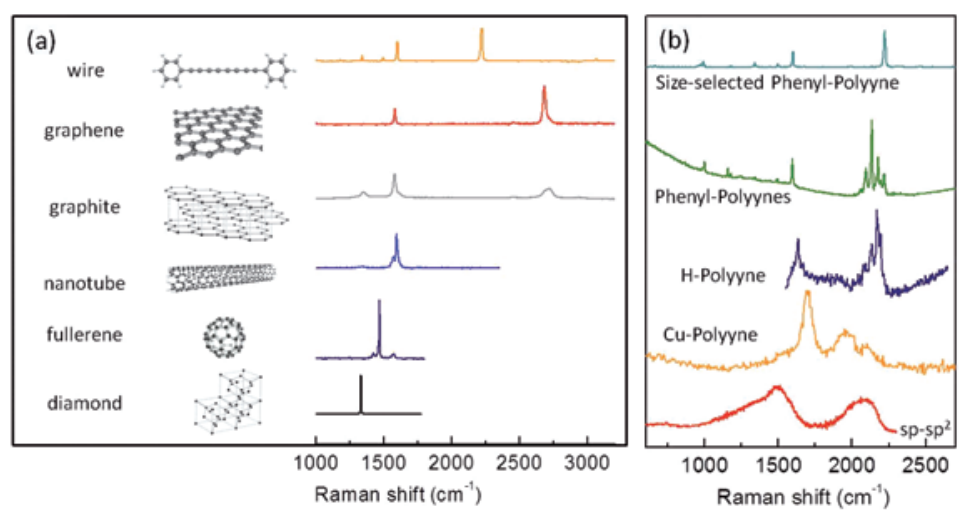

Fig. 6 - (a) Raman spectra of graphite, diamond and carbon nanostructures (fullerene C60, single wall carbon nanotubes, graphene and phenyl-polyynes).

(b) Raman spectra of different type of wires. 
Stability can be easily studied by Raman spectroscopy as one of the major problems arising when dealing with sp carbon wires. A part from systems with suitable terminations, the majority of sp carbon suffers from a poor stability. What typically happens is a sp-to-sp ${ }^{2}$ transition due to cross-linking reactions. To this problem, a suitable strategy is the use of bulky terminating groups able to prevent cross-linking due to steric hindrance. An alternative route to the direct synthesis of stable structures is the stabilization of already formed wires. For instance $\mathrm{H}$-terminated polyynes can be embedded in a solid assembly of $\mathrm{Ag}$ nanoparticles, resulting in a sample stable for several weeks at room temperature and in air conditions. The stabilization steps can be followed by Raman spectroscopy, as shown in Fig. 7.[50] More recently Hayashi and co-workers produced a polymeric composite (i.e. poly(vinyl alcohol)) containing polyynes stabilized by Ag nanoparticles. [51]

The stability has been also studied, in particular the cumulene/polyyne stability, the sp-to-sp ${ }^{2}$ transition in different conditions and the variation of length distribution when interacting with metal nanoparticles.[28,29,52,53]
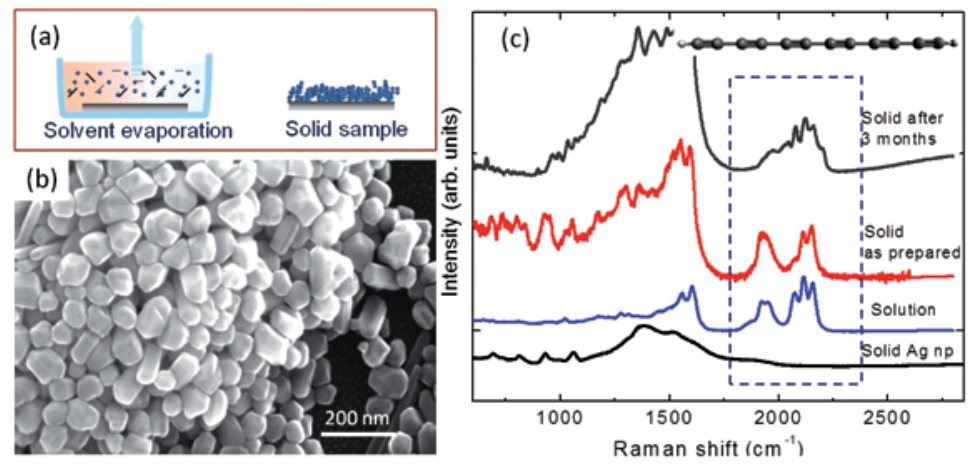

Fig. 7 - (a) Schematic process of stabilization of H-polyynes.

(b) Scanning electron microscope image of the silver nanoparticle assembly and (c) Raman spectra of the solid silver assembly alone, the solution of mixed polyynes and nanoaprticles, the solid assembly with polyynes as prepared and the same sample after 3 months showing that the signal from sp carbon is still present.

Actually, the interaction with metal nanoparticles can be exploited to increase the Raman signal by means of surface enhanced Raman scattering (SERS). Metal nanoparticles have collective excitations of the surface conduction electrons (i.e. surface plasmons) that depend on the par- 
ticle size. If the surface plasmon absorption matches the incident laser wavelength used for Raman, the high electromagnetic field close to the particle can enhance the Raman signal for order of magnitudes. SERS is usually employed to detect very small amount of sample, in the best conditions single molecule spectroscopy has been reported.[54] Two are the mechanisms involved in the SERS process: electromagnetic and chemical effect. The former is the due to the local enhancement of the electric field while the latter is active when some chemical interaction of the sample with the SERS active medium (i.e. nanoparticles) takes place. Carbon atomic wires in solution can be studied by SERS, the procedure is based on mixing the sample solution (H-terminated polyynes) with noble metal nanoparticles in solution (e.g. silver or gold colloids) as shown in Fig. 8. Silver colloids with diameters in the 20-40 nm range show an absorption peak (surface Plasmon) at about $420 \mathrm{~nm}$ while after mixing the Plasmon absorption is extended down to the infrared region (see Fig. 8b,e). This is an indication of a chemical effect in which polyynes aggregate when interacting with metal nanoparticles and allows to perform SERS with different excitation wavelengths.[52] The SERS effect is shown in Fig. 8c, where the comparison between Raman and SERS indicates an enhancement factor of about one million times.
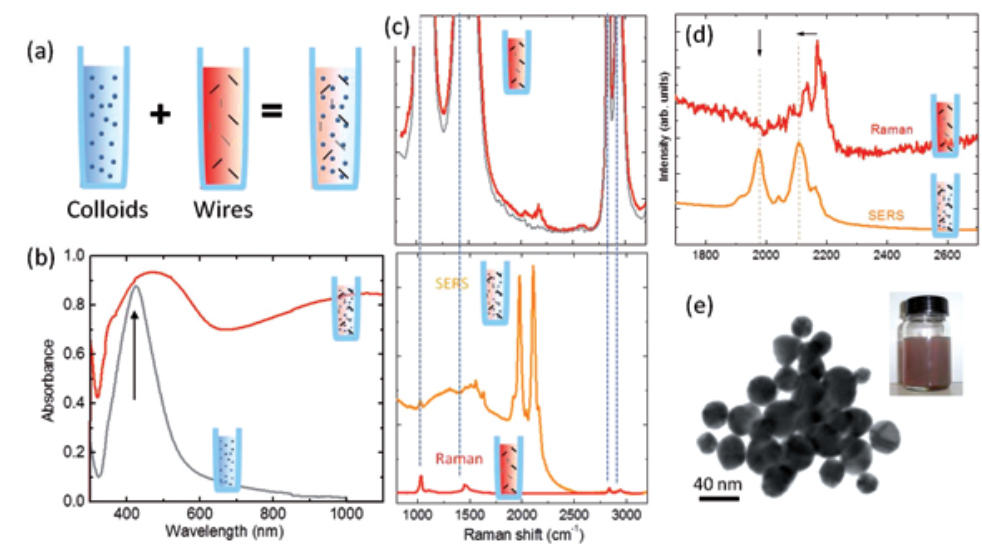

Fig. 8 - Raman and SERS of H-terminated polyynes. (a) Scheme of SERS experiment; (b) UV-vis absorption spectra of silver nanoparticles; (c) Raman and SERS of polyynes:

Raman spectrum compared with the spectrum of the solvent alone (top) and SERS compared with Raman where the peaks from the solvent are used for normalization (bottom); (d) particular of the SERS and Raman spectra in the sp-carbon spectral region and (e) scanning electron microscopy image of Au nanoparticles as a SERS active medium. 
A closer inspection to the SERS and Raman spectra reveals that the two spectra are not the same. In addition to the strong enhancement it is possible to observe a redshift of the main Raman peak together with the appearance of a new feature at lower wavenumbers (below $2000 \mathrm{~cm}^{-1}$ ). With the support of numerical calculations it is possible to explain such changes by a charge transfer from the nanoparticle to the wire. One electron moves to the wire and this induces a rearrangement of the structure with a strong reduction of the BLA.[48] The change in the BLA is responsible for the modification of the SERS spectrum with respect to the Raman one. Wire modification due to charge transfer is an interesting effect that can be used to tune the properties of the wires. In fact reduction of the BLA means a tendency towards a cumulene-like structure driven by interaction with noble metal particles. Such mechanism was observed not only in $\mathrm{H}$-terminated polyynes but also in other systems such as phenyl-polyynes thus opening a way for the external tuning of wire structure, without the need to change the termination to induce cumulene-like character.

\section{TOWARDS APPLICATIONS}

Carbon atomic wires have a great potential interest for technological applications. The possibility to tune the properties opens the way to novel functional systems to be used as building blocks for advanced materials and devices. Wires may be employed in a solution-processable approach with low cost and environmental friendly techniques. In this context the availability of metallic, semiconducting and insulating properties in the same system and the possibility to tune the energy and optical gap can overcome some problems in molecular and flexible electronics.

Appealing electron transport properties are expected for these wires as outlined by a number of theoretical investigations.[55,56] A pioneer work has been done by Lang and Avouris who computed the conductance of a wire connected to metal electrodes and outlined an oscillatory behavior depending on the even or odd number of carbon atoms. $[57,58]$ In particular, cumulenes as 1-D metals are expected to display ballistic conductance, a process where electrons move without scattering, that results in a constant conductance, not depending on 
the wire length, at the value of $2 \mathrm{G}_{0}$ where $G_{0}=2 e^{2} / b=77 \mu S$ (equivalent to a resistance of about $12 k \Omega$ ) is the quantum of conductance.[59] A very few papers recently reported the measurement of the conductivity of single wires. O. Cretu et al. measured the conductivity of a wire connecting two graphene flakes in the transmission electron microscope used for the top-down fabrication of the wire, as shown in Fig. 9a-c. Other groups used polyynes suspended between a metal surface and the metal tip of a scanning tunneling microscope as the contact electrodes. It was observed a larger resistance than the expected value, this is possibly due to the contact resistance between the wire and the electrodes.[60,61] Other considered systems include wires connecting graphene sheets and carbon nanotubes. In the latter case Yuzvinsky et al. reported the observation of a non monotonic current-voltage (I-V) curve with an apparent negative differential resistance (i.e. the current decreases when increasing the applied voltage).[62] Such behavior can be explained by invoking the possibility of a resonant tunneling behavior in which electrons go through the contacts by means of a quantum tunneling process. A memory device has been proposed based on the formation and disruption of a wire across fractured graphene which results is a change in the conductivity, as shown in Fig. 9d-f.[63] The wire formation and disruption process is repeatable and is controlled through the voltage applied on the two sides of the device. In such a device the reported conductance seems to match very well with a ballistic transport regime showing a characteristic staircase behavior in the I-V curve (see Fig. 9f). A similar device was proposed by $\mathrm{Li}$ et al. even though a nanoelectromechanical mechanism was considered responsible for the change in conductivity and only in a note the authors refer to the possibility of wire formation. [64]

One of the major problems arising in these proposed devices is that the wire structure (cumulene/polyyne) is poorly controlled or even not known. In this framework it has to be noticed that structural rearrangements of polyynes towards a more equalized structure (i.e. cumulene-like) occurring upon external perturbations such as UV light irradiation or charge transfers after interaction with metal nanoparticles have been recently observed by IR and surface enhanced Raman spectroscopy (SERS).[31,48] To this regard the manipulation of the structure of wires by external signals (optical and/or electrical) is a new and exciting challenge. 


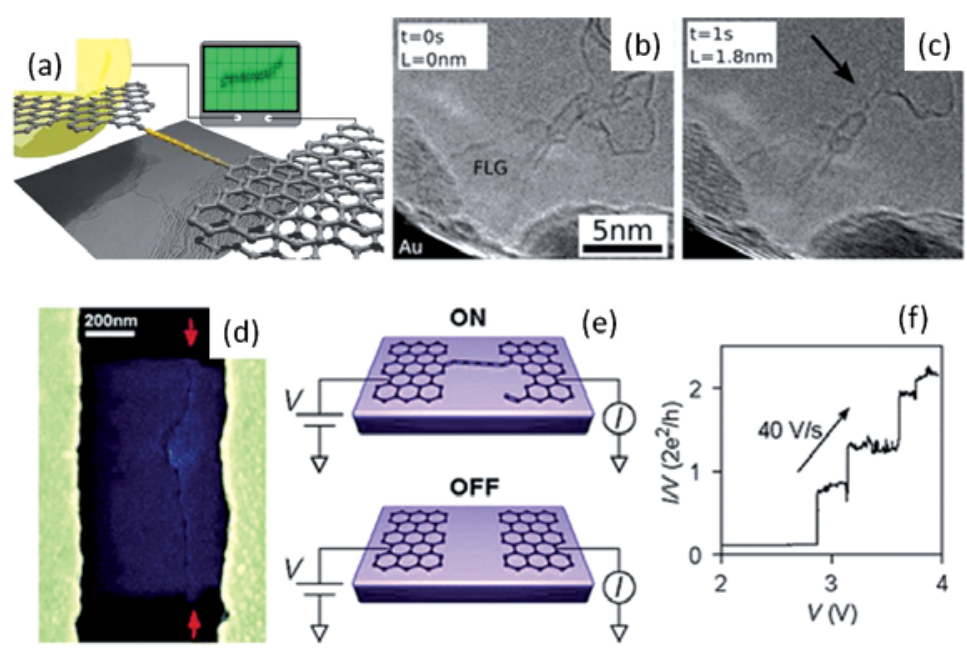

Fig. 9 - (a-c) Electrical measurements of a carbon atomic wire connecting two graphene edges. Reprinted (adapted) with permission from (B. Standley, W. Bao, H. Zhang Nano Letters 8, 3345 (2008)). Copyright (2008) American Chemical Society. (d-f) Memory device based on the formation and disruption of wires across graphene. Reprinted (adapted) with permission from (O. Cretu, A.R. Botello-Mendez, I. Janowska et al. Nano Lett. 13, 3487-3493 (2013)). Copyright (2013) American Chemical Society.

\section{CONCLUSIONS AND PERSPECTIVES}

The science of carbon nanostructures is a wide field embracing physics, chemistry, materials science and nanotechnology. Carbon nanostructures are amongst the largest investigated systems in the last three decades, as witnessed by the literature on fullerenes, nanotubes and graphene. In this framework there is still space for novel systems and allotropes, one possibility is represented by carbon atomic wires, linear 1-dimensional systems made by sp-hybridized carbon atoms, as discussed in this paper. Such systems are appealing for fundamental studies related with the formation of carbon clusters (the so-called fullerene road), with quantum effects in 1-dimensional systems and for applications due to the tunability of their functional properties. Carbon atomic wires, though with some difficulties, can be nowadays synthesized with different techniques in different forms (length and termination), characterization techniques such as Raman spectroscopy allow to 
detect the structure of the wires and recently the feasibility of measuring the electrical properties down to the single wire has been demonstrated, together with the realization of nano-scale devices. In this context carbon atomic wires have the potential to play a role as novel carbon nanostructures where a lot of work is still to be done to understand the properties and to synthesize systems with tailored properties. Very recently Yakobson and co-workers have underlined the mechanical properties that are expected, from theoretical predictions, to outperform graphene and nanotubes.[65] Moreover they show that cumulene structures are stabilized by vibrations and mechanical strain plays a key role in inducing a metal-to-insulator transition thus opening new opportunities for electromechanical switching and conductive polymer applications. [66] Theoreticians have been also attracted by hybrid sp$\mathrm{sp}^{2}$ systems in the form of 2-dimensional layers, the chief example being graphyne. Simulations have shown that such systems can have very interesting electronic properties such as an electron mobility even higher than graphene as reported by Malko et al..[67] The synthesis of such systems in extended form is still to be developed while the chemical synthesis as large molecules have been reported by Diederich and coworkers. [68] The quest for new carbon forms is still open, researchers have been looking for carbon atomic wires sp-hybridized carbon systems between the stars then to land in the nano-world where atomic wires and novel hybrid allotropes as 2 -dimensional sp-sp ${ }^{2}$ systems still wait to be studied, developed in detail and maybe applied in advanced technologies.

\section{ACKNOWLEDGEMENTS}

C.S. Casari would like to acknowledge C.E. Bottani, G. Zerbi, P. Milani and C. Castiglioni for useful discussion and M. Tommasini, A. Milani, A. Lucotti, V. Russo, A. Li Bassi, L. Ravagnan, C. Lenardi, P. Piseri, F. Siviero, for the work done in collaboration from 2000 to present. 


\section{REFERENCES}

[1] A. Hirsch. Nature Mat. 9, 868 (2010).

[2] S. Tennant. Phil. Trans. of the Roy. Soc. of London, 87, 123-127(1797).

[3] A. El Goresy and G. Donnay. Science 161, 363 (1968).

[4] A.G. Whittaker, P.L. Kintner. Science 165, 589 (1969).

[5] A.G. Whitteker. Science 200, 763 (1978).

[6] Carbyne and Carbynoid structures, R.B. Heimann, S.E. Evsyukov, L. Kavan Eds, Kluwer 1999.

[7] P.P.K. Smith, P.R. Buseck. Science 216, 984 (1982).

[8] A.G. Whittaker. Science 229, 485 (1985).

[9] P.P.K. Smith, P.R. Buseck. Science 229, 486 (1985).

[10] H. Kroto. Nobel Lecture December 71996 (http://www.nobelprize.org)

[11] R.J. Lagow, J.J. Kampa et al. Science 267, 362 (1995).

[12] T. Wakabayashi, A.-L. Ong et al. J. Phys. Chem. B 108, 3686-3690 (2004).

[13] L. Ravagnan, T. Mazza et al. Chemical Communications 47, 2952-4 (2011).

[14] A.A. Zaidi, A. Hu, M.J. Wesolowski et al., Carbon 48, 2517-2520 (2010).

[15] L. Ravagnan, F. Siviero et al. Physical Review Letters, 89, 285506 (2002).

[16] H. Tabata, M. Fujii and S. Hayashi. Carbon 44, 522-529 (2006).

[17] X. Zhao, Y. Ando, Y. Liu et al. Physical Review Letters 90, 187401 (2003).

[18] C. Jin, H. Lan, L. Peng et al. Physical Review Letters 102, 205501 (2009).

[19] Polyynes: Synthesis Properties and Applications, F. Cataldo Ed., Taylor \& Francis 2006.

[20] H. Tabata, M. Fujii, S. Hayashi et al. Carbon 44, 3168-3176 (2006).

[21] A. Lucotti, M. Tommasini, D. Fazzi et al. J. Am. Chem. Soc. 131, 4239-44 (2009).

[22] S. Szafert and J.A. Gladysz. Chemical Reviews 103, 4175-205 (2003).

[23] F. Cataldo, C.S. Casari. J. Inorg. Organ. Pol. and Mater. 17, 641-651 (2007).

[24] W.A. Chalifoux and R.R. Tykwinski. Nature Chemistry 2, 967-71 (2010).

[25] J.A. Januszewski, R.R. Tykwinski. Chem. Soc. Rev. 43, 3184-3203 (2014).

[26] F. Cataldo. J. of Inorg. and Organomet. Polym. and Mater. 16, 15 (2006).

[27] V. Cadierno and J. Gimeno. Chemical Reviews 109, 3512-60 (2009).

[28] L. Ravagnan, P. Piseri, M. Bruzzi et al. Physical Review Letters 98, 216103 (2007).

[29] C.S. Casari, A. Li Bassi, L. Ravagnan et al. Physical Review B, 69, 075422 (2004).

[30] C.S. Casari, A. Li Bassi, A. Baserga et al. Physical Review B 77, 195444 (2008).

[31] M.M. Yildizhan, D. Fazzi et al. Journal of Chemical Physics 134, 124512 (2011).

[32] R.E. Peierls, Quantum Theory of Solids (Clarendon, Oxford, 1955), p. 108.

[33] A. Milani, M. Tommasini et al. Physical Review B 74, 153418 (2006).

[34] A. Milani, M. Tommasini, G. Zerbi. J. of Chem. Phys. 128, 064501 (2008).

[35] A. Milani, M. Tommasini, G. Zerbi. J. of Raman Spectroscopy 40, 1931-1934 (2009).

[36] L. Zhang, H. Ma, N. Yao et al. J. Vac. Sci. Tech. 25, 545 (2007). 
[37] A. Hu, S. Griesing, M. Rybachuk et al. J. of Applied Physics 102, 074311 (2007).

[38] L. D'Urso, G. Compagnini, O. Puglisi et al. J. of Phys. Chem. C 111, 17437 (2007).

[39] G. Compagnini, S. Battiato, O. Puglisi et al. Carbon 43, 3025-3028 (2005).

[40] A. Hu, M. Rybachuk, Q.-B. Lu et al. Appl. Phys. Lett. 91, 131906 (2007).

[41] A. Hu, Q.-B. Lu, W.W. Duley, M. Rybachuk. J. Chem. Phys. 126, 154705 (2007).

[42] F. Cataldo. Carbon 41, 2671 (2003).

[43] F. Cataldo. Tetrahedron 60, 4265-4274 (2004).

[44] Phonons, Theory and Experiments II, P. Brüesch Springer 1982.

[45] A.C. Ferrari, D.M. Basko. Nature Nanotechnology 8, 235 (2013).

[46] A.C. Ferrari et al. Phys. Rev. Lett. 97, 187401 (2006).

[47] F. Innocenti, A. Milani, C. Castiglioni. J. Raman Spectrosc. 41, 226-236 (2010).

[48] A. Milani, A. Lucotti, V. Russo et al. J. of Phys. Chem. C, 115(26), 12836 (2011).

[49] L. Ravagnan, N. Manini, E. Cinquanta et al. Phys. Rev. Lett. 102, 245502 (2009).

[50] C.S. Casari, V. Russo, A. Li Bassi et al. Applied Physics Letters 90, 13111 (2007).

[51] S. Okada, M. Fujii, S. Hayashi. Carbon 49, 4704-4709 (2011).

[52] A. Lucotti, M. Tommasini, M. Del Zoppo et al. Chem. Phys. Lett. 417, 78-82 (2006).

[53] A. Lucotti, C.S. Casari, M. Tommasini et al. Chem. Phys. Lett. 478, 45-50 (2009).

[54] K. Kneipp, Y. Wang, H. Kneipp et al. Phys. Rev. Lett. 78, 1667 (1997).

[55] S. Tongay, R. Senger, S. Dag, and S. Ciraci. Phys. Rev. Lett. 93, 136404 (2004).

[56] Z. Zanolli, G. Onida, J.-C. Charlier. ACS Nano 4, 5174-80 (2010).

[57] N.D. Lang and Ph. Avouris. Phys. Rev. Lett. 81, 3515-3518 (1998).

[58] N.D. Lang and Ph. Avouris. Phys. Rev. Lett. 84, 358 (2000).

[59] O. Cretu, A.R. Botello-Mendez, I. Janowska et al. Nano Lett. 13, 3487-3493 (2013).

[60] P. Moreno-García, M. Gulcur et al. J. Am. Chem. Soc. 135, 12228-12240 (2013).

[61] F. Chen, X. Li, J. Hihath et al. J. Am. Chem. Soc. 128, 15874-15881 (2006).

[62] T.D. Yuzvinsky, W. Mickelson, S. Aloni. Nano Letters 6, 2718-22 (2006).

[63] B. Standley, W. Bao, H. Zhang. Nano Letters 8, 3345 (2008).

[64] Y. Li, A. Ainitskii, J.M. Tour. Nature Materials 7, 966 (2008).

[65] M. Liu et al. ACS Nano 7, 10075-10082 (2013).

[66] V.I. Artyukhov et al. Nano Lett. 14, 4224-4229 (2014).

[67] D. Malko, C. Neiss. Phys. Rev. Lett. 108, 086804 (2012).

[68] F. Diederich, M. Kivala. Adv. Mater. 22, 803-812 (2010). 
